Letters to the Editor

\section{Mixed bacterial endocarditis in an intravenous drug misuser}

Sir,

Bacterial endocarditis is a frequent complication of intravenous (i.v.) drug misuse often associated with a significant mortality and morbidity. Staphylococcus aureus is the commonest infecting organism, usually presenting as a 'pneumonia' or septicaemia and with no or minimal cardiac murmurs.' However, other pathogens, especially skin derived streptococci, are important and serious causes of endocarditis in this group of patients. In fact, $\beta$-haemolytic streptococci Group $\mathbf{G}$ produce a highly invasive and destructive endocarditis associated with a $36 \%$ mortality. $^{2}$

Drug users admitted to hospital with clinical features suggestive of endocarditis are often commenced on antimicrobial therapy on a best guess basis. The following case, which illustrates an uncommon mixed infection, highlights the importance of initially using a combination of benzylpenicillin in high doses, flucloxacillin and an aminoglycoside, until culture and sensitivity results are available, to ensure that broad cover effective therapy is instigated early.

A 29 year old intravenous drug user was admitted with a one week history of fever, rigors, headaches, pleuritic chest pain and a cough productive of purulent sputum. His temperature was $39^{\circ} \mathrm{C}$. He had a loud systolic murmur maximal at the left sternal edge and bilateral basal pleural rubs. Echocardiography did not reveal any vegetations (as in up to $70 \%$ of cases of infective endocarditis). ${ }^{3}$

A provisional diagnosis of a right sided bacterial endocarditis was made. Empirical therapy with flucloxacillin and gentamicin was commenced. Twenty four hours following admission coagulase positive Staphylococcus aureus was isolated in two sets of cultures. A further 48 hours later beta haemolytic streptococcus (serotype group G) was also isolated. At this stage there was evidence of gentamicin-induced nephrotoxicity despite monitoring of serum levels. The regime was changed to i.v. benzylpenicillin, ciprofloxacin and flucloxacillin. Penicillin and the latter combination were found to be satisfactorily bacteriocidal to the streptococcus and staphylococcus respectively. Regrettably the patient took his own discharge 18 days after admission.

As this case shows, drug using patients are notoriously difficult to treat as inpatients for the recommended period of 4 to 6 weeks. Recently, a shorter two week regime using nafcillin-tobramycin was shown to be effective. ${ }^{4}$

Finally there is increasing evidence that ciprofloxacin may be an effective alternative treatment for staphylococcal endocarditis. ${ }^{5}$ This may allow treatment to be continued on an outpatient basis in those patients refusing to remain in hospital. It does not require regular monitoring of therapeutic levels, which is an added advantage as drug users usually have poor venous access.

$$
\begin{array}{r}
\text { Dilip Nathwani, } \\
\text { Dermot H. Kennedy } \\
\text { Department of Infection and Tropical Medicine, } \\
\text { Ruchill Hospital, Bilsland Drive, } \\
\text { Glasgow G20,UK. }
\end{array}
$$

\section{References}

1. Report of a Working Party of the British Society for Antimicrobial Chemotherapy. Antibiotic treatment of streptococcal and staphylococcal endocarditis. Lancet 1985, ii: 815-817.

2. Smyth, E.G., Pallett, A.R. \& Davidson, R.N. Group G streptococcal endocarditis: two case reports, a review of the literature and recommendations for treatment. $J$ Infection 1988, 16: 169-176.

3. Wann, L.S., Dillon, J.C., Weyman, A.E. \& Feigenbaum, H. Echocardiography in bacterial endocarditis. $N$ Engl $J$ Med 1976, 295: 135-139.

4. Chambers, H.F., Miller, R.T. \& Newman, M.D. Right-sided Staphylococcus aureus endocarditis in intravenous drug abusers: two week combination therapy. Ann Intern Med 1988, 109: 619-624.

5. Fernandez-Guerrero, M., Rouse, M., Henry, N. \& Wilson, W. Ciprofloxacin therapy of experimental endocarditis caused by methicillin susceptible or methicillin resistant Staphylococcus endocarditis. Antimicrob Agents Chemother 1988, 32: 747-751.

\section{High dose atropine in organophosphorus poisoning}

Sir,

We would like to report a case of organophosphorus poisoning treated with a very high dose of intravenous atropine. A 24 year old female was admitted following ingestion of $50 \mathrm{ml}$ of malathion as a suicidal attempt. She developed abdominal pain, vomiting, breathlessness and altered sensorium over a period of 2 hours. Examination revealed a comatosed patient with pin point pupils. She was in acute respiratory distress and cyanosed. All investigations including haemoglobin, total leucocyte count, ESR, blood urea, serum creatinine, electrolytes, liver functional tests, and cerebrospinal fluid were normal. Plasma cholinesterase activity was reduced to $30 \%$. She was immediately intubated and put on intermittent positive pressure ventilation. Intravenous atropine $2 \mathrm{mg}$ every 5-10 minutes and pralidoxime $1 \mathrm{~g}$ every 8 hours were started, but the patient did not respond. Subsequently, the dose of atropine was increased to $6 \mathrm{mg}$ at every 10 minutes with no signs of atropine toxicity. The patient remained on a ventilator for 8 days and received a total of $3369 \mathrm{mg}$ of intravenous atropine. She made a complete recovery.

Malathion, a highly toxic organophosphorus compound most widely used as an insecticide, is a common cause of poisoning in India. ${ }^{1}$ The toxicity occurs as a result of ingestion, inhalation or by absorption through skin. ${ }^{2}$ The recommended dose of atropine is $2 \mathrm{mg}$ every 5-10 minutes till the muscarinic effects due to toxicity are reversed. Atropine in such a high dose as given in this patient has not been reported so far. The reason why such a high dose of atropine was needed could not be explained.

S. Afzaal,

A. Shakoor,

M.U. Rabbani,

Shadab A. Khan

Departments of Medicine and Anaesthesia, Jawaharlal Nehru Medical College. Aligarh Muslim University,

Aligarh

India. 


\section{References}

1. Sigh, S., Sharma, B.K., Wahi, P.L., Anand, B.S. \& Chugh, K.S. Spectrum of acute poisoning in adults (10 years experience). J Assoc Physicians India 1984, 32: 561-563.

2. Kipling, R.M. \& Cruickshank, A.N. Organophosphate insecticide poisoning. Anaesthesia 1985, 40: 281-284.

\section{Subacute cor pulmonale due to tumour embolization to the lungs}

Sir,

Although metastatic spread of tumour to the lungs is common, subsequent production of cor pulmonale is not. Since the first description of subacute cor pulmonale caused by multiple tumour emboli to the lungs in 1937, no more than 40 cases have been reported, five of which were related to breast neoplasms.' The purpose of this letter is to report a new case. We emphasize the value of the pulmonary microvascular cytology study in the diagnosis of this entity.

A 39 year old woman was admitted with a one week history of rapidly progressive breathlessness. Twelve months before admission, a left modified radical mastectomy was performed for infiltrating ductal carcinoma. Chemotherapy with cyclophosphamide, methotrexate and fluorouracil was administered for the next 6 months. Physical examination disclosed a respiratory rate of $30 / \mathrm{min}$ at rest and one node on the left side of her neck. Initial arterial blood gases were: $\mathrm{PaCO}_{2} 3.7 \mathrm{kPa}, \mathrm{PaO}_{2}$ $6.9 \mathrm{kPa}$ and $\mathrm{pH}$ 7.5. Her chest radiograph was normal. A perfusion lung scan demonstrated no abnormalities. Swan-Ganz catheterization revealed the following pressures $(\mathrm{mmHg})$ : right atrial, 15 ; right ventricular, $65 / 12$; pulmonary artery, $60 / 45$; and pulmonary capillary wedge, 4. Cytological examination of blood obtained via floating pulmonary artery catheter in the wedged position ${ }^{2}$ revealed both malignant cells and megakaryocytes. Cervical lymph node biopsy specimen contained tumour. Despite maximum supportive measures and chemotherapy with doxorubicin and cyclophosphamide, she died on the fourth hospital day. Autopsy was denied.
Subacute cor pulmonale is a distinct disease entity but is often misdiagnosed. Although the diagnosis in our patient was supported on the basis of both Swan-Ganz catheterization and pathological lymph node findings, it was confirmed after pulmonary microvascular cytology examination.

The diagnosis of lymphangitic spread of carcinoma in the lungs is sometimes difficult. In a recent study, ${ }^{3}$ malignant cells were found on pulmonary microvascular cytology samples in seven of eight patients studied in whom lung biopsy or autopsy demonstrated that lymphangitic carcinomatosis was the primary cause of their respiratory distress. In 17 patients with active cancer but who had no evidence of pulmonary metastasis, in all of these patients except one, pulmonary microvascular cytology showed megakaryocytes and no other abnormalities.

As Masson et al. conclude, ${ }^{3}$ this technique may be valuable when lung biopsy is refused or is thought to be hazardous.

\author{
Jose Luis Rodriguez-Garcia \\ Juan Carlos Hernando \\ Manuel Serrano \\ Miguel Angel Aguinaga' \\ Luis Escribano ${ }^{2}$ \\ Services of Medicine, Intensive Care \\ and ${ }^{2}$ Haematology, \\ Hospital Ramón y Cajal, \\ Carretera de Colmenar km 9,100, \\ 28034 Madrid, Spain.
}

\section{References}

1. Xian-wu, He, Yi-hai, Tang, Zhong-qiang, Luo, Liang-di, Gong, Tsung, $\mathbf{O}$, Cheng. Subacute cor pulmonale due to tumor embolization to the lungs. Angiology, 1989, 40: 11-17.

2. Masson, R.G. \& Ruggieri, J. Pulmonary microvascular cytology. A new diagnostic application of the pulmonary artery catheter. Chest 1985, 88: 908-914.

3. Masson, R.G., Krikorian, J., Lukl, P., Evans, G.L. \& McGrath, J. Pulmonary microvascular cytology in the diagnosis of lymphangitic carcinomatosis. N Engl J Med 1989, 321: $71-73$. 